\title{
Dynamic Analysis and Control of Biochemical Reaction Networks
}

Irene Otero-Muras ${ }^{1}$, Gábor Szederkényi ${ }^{2}$, Katalin M. Hangos ${ }^{2}$, Antonio A. Alonso ${ }^{1 *}$

Corresponding author: antonio@iim.csic.es

1. Process Engineering Group,Instituto de Investigaciones Marinas-CSIC.

C/Eduardo Cabello, 6 - 36208 Vigo, Spain, Phone: +34 986231930, Fax: +34 986292762

2. Process Control Research Group, Computer and Automation Research Institute, Hungarian Academy

of Sciences, H-1518 Budapest, P.O. Box 63, Hungary Phone: +36 12796000, Fax: +36 14667503

\begin{abstract}
In the present work, we combine concepts and tools from Irreversible Thermodynamics and Control Theory in a contribution to unravel the origin of complex nonlinear behaviour in biochemical networks. Regarding cells as thermodynamic systems, we can consider dynamic evolution of intracellular processes in terms of the combined action of an endogenous entropy production and the entropy flux associated to chemicals passing through the control volume. Based on a generalized description of biochemical systems, a physically motivated storage function is constructed and used for stability analysis. In this way, the entropy flux of open systems can be meaningfully modified by efficient nonlinear control schemes capable of network stabilization, and irreversible thermodynamics provide us with the physical insight to further interpret the controlled response.
\end{abstract}

Keywords: Biochemical Reaction Networks, Multiplicities, Passivity based control, Thermodynamics

\section{Introduction}

Biochemical networks consist of highly interconnected dynamic systems of chemical reactions interacting with the environment through mass and energy flows. Examples include metabolic or cell signalling pathways which are known to exhibit complex nonlinear behavior in the form of steady-state multiplicities, sustained oscillations or deterministic chaos. Complex dynamics of biochemical networks is a recurring theme in the literature due to its decisive role in physiology of living organisms [11]. As a relevant class of complex networks which conform the building blocks and circuitry upon which cells and living organisms operate [10] biochemical networks are receiving considerable attention from different scientific areas going from nonlinear physics to systems engineering [7]. Despite considerable progress in their dynamic aspects, the origin of biochemical complex nonlinear phenomena is still not always well understood and it can not be systematically predicted beyond a case by case basis. This hampers some biological processes being manipulated or monitored at the cell level. In this framework, efforts are needed to develop efficient and robust methods of stabilization and control.

In this paper, we present a systematic approach to the biochemical network dynamic analysis and control based on both thermodyamic and control theoretic tools. In the first instance (Section 1) the equations of the model for a general class of biochemical networks is established, setting the assumptions under which the results of the Chemical Reaction Network Theory [3] and Classical Kinetics [4] directly apply. The biochemical network is also rigorously tackled as a thermodynamic system interconnected with the environment by mass flows in order to further interprete the dynamics in terms of the entropy balance. This fact, on one hand, will provide us with the physical insight in the target to unravel the origin of complex behaviour (Section 3). On the other hand, the entropy balance will enable us to devise in a constructive manner efficient control schemes capable of robust network stabilization (Section 4). In this section we demonstrate also the underlying potential structure of a class of biochemical reaction networks to further use this property combined with passivity theory in developing stabilizing control laws. The results are illustrated through the paper using as an example a typical component of metabolic pathways: an enzyme-catalysed mechanism with substrate inhibition. 


\section{A general class of biochemical reaction networks}

The systems under study are those metabolic, genetic and signaling networks in which pathway stoichiometry is known, and the following assumptions are considered:

i) the reaction rates of the kinetic mechanisms obey the mass action law,

ii) the spatial distribution of products can be neglected,

iii) the process takes place in isothermal conditions.

Under these assumptions, a biochemical reaction network is defined as a set of $n$ species or components $X_{i} \quad(i=1, \ldots, n)$ whose concentration or level of activity is a continuous variable $x_{i} \geq 0$, interacting among themselves through a set of reactions taking place in $r$ steps of the form:

$$
\sum_{i=1}^{n} \alpha_{i j} X_{i} \underset{k_{j}^{-}}{\stackrel{k_{j}^{+}}{\rightleftarrows}} \sum_{i=1}^{n} \beta_{i j} X_{i} \quad \text { for } j=1, \ldots r
$$

In (1), the integers $\alpha_{i j}$ and $\beta_{i j}$ are the stoichiometric coefficients for the component $X_{i}$ in the reaction step $j$, and the direct and inverse rates $W_{j}^{+}$and $W_{j}^{-}$respectively, are monomials of the concentrations of the species involved in the step $j$ according to the mass action law:

$$
W_{j}^{+}=k_{j}^{+} \prod_{i=1}^{n} x_{i}^{\alpha_{i j}} \quad W_{j}^{-}=k_{j}^{+} \prod_{i=1}^{n} x_{i}^{\beta_{i j}}
$$

where $k_{j}^{+}$and $k_{j}^{-}$are the constants of the direct and of the inverse rates. The expression (1) may represent different kind of kinetic mechanisms:

a) A reversible or equilibrium reaction if both constants of reaction are positive numbers.

b) An irreversible reaction if one of the constants is zero.

c) An input pseudo-reaction, an irreversible step for which all the stoichiometric coefficients $\alpha_{i j}=0$.

d) An output pseudo-reaction, an irreversible step for which all the stoichiometric coefficients $\beta_{i j}=0$.

The dynamic evolution of the network, which state space is represented by the vector $x$ of the component concentrations is described by a set of ordinary differential equations of the form:

$$
\frac{d x}{d t}=f(x, k)
$$

where $f$ is a nonlinear function in which only mass action kinetics kind of monomials are allowed, and $k$ refers to internal or external conditions held constant during the dynamic process [9], i.e. to the monomial constants in (2). The reaction networks that, in addition to be isolated from the environment, are endowed with the property of weakly reversibility are considered closed reaction systems in the thermodynamic sense. By means of a suitable selection of the boundary of a virtual domain or control volume, a biochemical reaction network may be partitioned in two subnetworks: a conservative and weakly reversible inner subnetwork (closed reaction system) and a subnetwork representing the environment connections, including as well the irreversible steps such as matter generation or extinction, that are in fact input/output flows through the virtual domain. 


\subsection{Review of the Chemical Reaction Network Theory formalism}

The Chemical Reaction Network Theory [6] developed by Horn, Jackson and Feinberg provides a framework for the study of the dynamics of chemical reactors. A reaction network defines a directed graph on the so called complexes, that are the multisets of chemical species that appear on the left and right hand sides of each reaction step in (1). Each complex $c_{k}$ has associated a vector $y_{k}$ representing the stoichiometry of each specie in the complex as follows:

$$
c_{k}=\sum_{i=1}^{n} y_{i k} X_{i} \quad y_{k} \in \mathbb{P}^{n}
$$

where $y_{i k}$ is related with the stoichiometric coefficients $\alpha_{i j}$ or $\beta_{i j}$ in (1) depending on whether $k$ is a reactive or product complex. The $n$-dimensional vector $y_{k}$ is the $k$-th column of the so called stoichiometric matrix $Y$ that maps the $n$ dimensional space of the species on the $m$ dimensional space of the complexes. Each of the $m$ complexes of the network, that corresponds to a node in the graph, has associated the nonlinear part of a monomial in (2), so it is posible to define the following $m$-dimensional vector of monomials:

$$
\psi(x)=\sum_{y} x^{y} \quad \psi_{k}=\prod_{k=1}^{n} x_{k}^{y_{i k}}
$$

The arrows in the graph stand for irreversible reactions. The arrow which connects the nodes $i$ and $j$ is the reaction $c_{i}-c_{j}$ in which complex $i$ converts in complex $j$. The weights associated to the arrows are the corresponding positive constants in (2), that constitute the entries of the adjacency matrix $R$ of the graph.

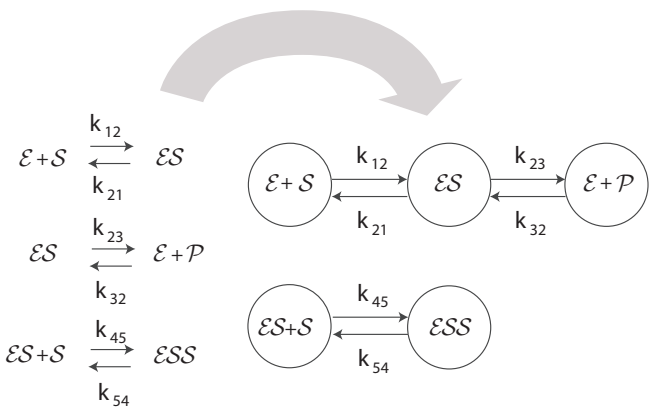

(a)

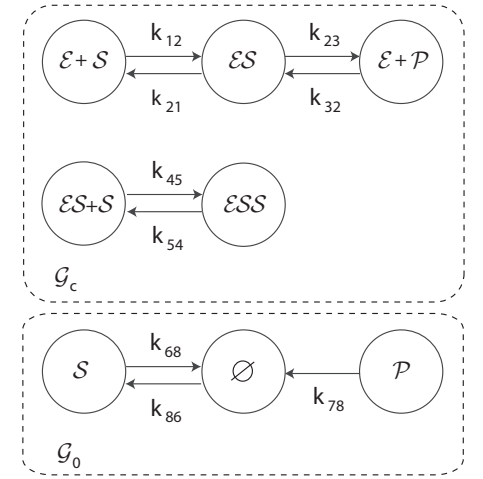

(b)

Figure 1. Michaelis Menten kinetics mechanism. Graphical description of the closed (a) and open (b) models

Attending to the connectivity properties, the so called linkage classes and weakly reversible linkage classes in (3) have an equivalence in graph theoretic terms in the components and strongly connected components, respectively. The dynamics (3) is derived from the graphical structure as follows:

$$
\dot{x}=Y \cdot A_{k} \cdot \psi(x)
$$

where $Y$ is the stoichiometric matrix which columns are the vectors $y_{k}$ associated to the complexes, $\psi(x)$ is the vector of monomials (5) and $A_{k}$ is the matrix settled starting from the adjacency matrix $R$ of the graph:

$$
A_{k}=R-\operatorname{diag}\left(R^{T} \cdot \overrightarrow{1}\right)
$$


Each of the reactions $c_{i}-c_{j}$ has associated a vector $y_{i}-y_{j}$. The so called stoichiometric subspace $S$ is the span of the set of reaction vectors [3]. Some important results on the stability of reaction networks provided by the Chemical Reaction Network Theory are stated in terms of the deficiency, that is defined by the formula: $\delta=m-\ell-s$ where $m$ is the number of complexes of the network, $\ell$ is the number of linkage classes and $s$ is the dimension of the aforementioned stoichiometric subspace. The Deficiency Zero Theorem [3] asserts that a weakly reversible network of deficiency zero has an unique and asymptotically stable steady state in each reaction simplex (see subsection 2.3) for arbitrary values of the constants $k$ in (3). According to our formalism, thermodynamically closed reaction networks are deficiency zero. In order to cope with the open systems description, a zero complex representing the environment is introduced, and the outflow or inflow of a component from/to the control volume is modelled by a pseudo reaction between the involved specie and the zero complex as it can be seen in Fig. 1b. Starting from a closed system, the dynamics (6) varies as we open the system as a result of being the matrices $Y, A_{k}$ and the vector $\psi$ modified by the introduction of the new complexes in the network [3]. In this contribution, we propose an alternative representation which derived from the graphical description factorizes (6) into an inner (closed reaction subnetwork) term and an environment connection as follows:

$$
\dot{x}=Y \cdot A_{k c} \cdot \psi_{c}(x)+\Phi \cdot Y \cdot A_{k o} \cdot \psi_{o}(x)
$$

where the subindex $c$ refers to the subgraph which represents a closed system of deficiency zero and the subindex $o$ denotes the input-output partition of the graph. For a closed system the parameter $\Phi$ vanishes.

\subsection{Review of the classical kinetic formalism}

Assuming that all the steps (1) are reversible, we can adopt the classical kinetic formalism described in [4] for the dynamics (3). The reaction rate for each step can be expressed in a polynomial form (2) as : $W_{j}=W_{j}^{+}-W_{j}^{-}$. Accordingly, the set of ordinary differential equations that describe the dynamics of the network (3) can be written in the following compact matrix form:

$$
\dot{x}=\mathcal{N} \cdot W(x), \quad \dot{x}_{i}=\sum_{j=1}^{r} \nu_{i j}\left(W_{j}^{+}-W_{j}^{-}\right)
$$

where $\mathcal{N}=\left[\nu_{i j}\right]$ is the $n \times r$ coefficient matrix whose columns are the linearly independent stoichiometric vectors $\nu_{j}=\beta_{j}-\alpha_{j}$ being $\nu_{i j}=\alpha_{i j}-\beta_{i j}$ positive or negative depending on whether the specie $i$ is a product or a reactant in the reaction $j$ and $W(x) \in \mathbb{R}^{r}$ denotes the vector of reaction rates. The state-space representation of an open reaction system is constructed by adding a set of input and output terms to the closed reaction system (9). The set of ordinary differential equations governing the evolution of states in an open system becomes:

$$
\dot{x}=\mathcal{N} \cdot W(x)+\phi\left(x^{0}-x\right)
$$

where the parameter $\phi$ is related to the inverse of the residence time inside the control volume and $x^{0}$ represents the inflow concentration vector. It should be noticed at this point the equivalence between expressions (10) and (8). Typical phenomena in biochemical networks like degradation and generation of components, for example, appear to be accommodated within these descriptions.

\subsection{The reaction simplex and the equilibrium manifold}

Mass conservation imposes the physical invariants of the dynamics (3) in a biochemical reaction network. The $n$ reacting species are composed by $p$ building blocks $\mathcal{C}_{k}(k=1, \ldots, p)$ that are not divided during 
the overall reaction, remaining their concentration constant. This fact gives rise in a closed system to the following set of algebraic equations corresponding to the $p$ mass conservation balances:

$$
\mathcal{C}_{k}^{0}=c_{k}^{T} \cdot x \quad \text { for } \quad k=1, \ldots, p
$$

where the entries of the vector $c_{k} \in \mathbb{N}^{n}$ represent the units of the building block $\mathcal{C}_{k}$ in each specie, and $\mathcal{C}_{k}^{0}=c_{k}^{T} \cdot x_{0}$ with $x_{0}=x(0)$. The intersection of the positive orthant (i.e. $\mathbb{R}_{+}^{n}$ ) with the set of $p$ conservation laws defines the so called reaction simplex for a given initial condition $x_{0}$ :

$$
\Omega\left(x_{0}\right)=\left\{x \in \mathbb{R}_{n}^{+} \mid c_{k}^{T}\left(x-x_{0}\right)=0, \quad k=1, \ldots, p\right\}
$$

which is invariant for the system dynamics. In the Classical Kinetic approach, a basis of $\operatorname{ker}\left(\mathcal{N}^{T}\right)$ determines the set of vectors $\left[c_{k}\right]$ in (12). In the Chemical Reaction Network Theory formalism, the reaction simplex is obtained as the intersection between the parallel to the linear variety defined by the stoichiometric subspace in which the initial concentration $x_{0}$ is contained, and the positive orthant. The reaction simplex is compact if and only if the system is conservative [3]. A thermodynamically closed system is a system that is weakly reversible and conservative, where the relationships $\dot{x}=0 \Leftrightarrow W\left(x^{*}\right)=0$ determine the set of equilibrium points $x^{*}$. We will show next by means of a Lyapunov argument that there exists an equilibrium point and it is unique for every initial condition belonging to the same reaction simplex. This is in agreement with the stability results derived from CRNT since thermodynamically closed reaction systems are of deficiency zero.

Proposition 1. The trajectories of an open network (10) with inlet concentration vector $x^{0}$ tend exponentially to the reaction simplex $\Omega\left(x^{0}\right)$.

Proof: Multiplying both sides in (10) by a basis of $\operatorname{ker}(\mathcal{N})$ we arrive to $c^{T} \dot{x}=\phi \cdot\left(c^{T} x^{0}-c^{T} x\right)$, that is, $\dot{\Omega}(x)=\phi \cdot\left(\Omega\left(x^{0}\right)-\Omega(x)\right)$. After integrating this last expression over time:

$$
\Omega(t)=\Omega\left(x^{0}\right)\left(1-e^{-\phi \cdot t)}\right)+\Omega\left(x_{0}\right) e^{-\phi t}
$$

so that $\lim _{t \rightarrow \infty} \Omega(t) \rightarrow \Omega\left(x^{0}\right)$ and convergence to the simplex can be concluded.

\subsection{Example: Michaelis Menten Kinetics with substrate inhibition}

2.4.1. The mechanism: The Michaelis Menten kinetics for the reaction $S \rightarrow P$ catalyzed by enzyme (where the substrate acts as an inhibitor) is described in biochemistry by means of a two dimensional state space system of equations:

$$
\frac{d P}{d t}=\frac{V_{\max }[S]}{K_{M}+[S]+\frac{[S]^{2}}{K_{i}}}, \quad \frac{d S}{d t}=-\frac{V_{\max }[S]}{K_{M}+[S]+\frac{[S]^{2}}{K_{i}}}
$$

where $[S]$ and $[P]$ represent the substrate and product concentrations, respectively. $V_{\max }$ is a constant term wich depends on kinetic factors and initial concentration of enzyme, and the constants $K_{M}$ and $K_{i}$ are the Michaelis and Inhibition constants respectively. As it is well known, the system described in (14) corresponds to a reduced order representation of the following mass action law based mechanism:

$$
E+S \underset{k_{1}^{-}}{\stackrel{k_{1}^{+}}{\rightleftarrows}} E S \quad E S \underset{k_{2}^{-}}{\stackrel{k_{2}^{+}}{\rightleftarrows}} E+P \quad E S+S \underset{k_{3}^{-}}{\stackrel{k_{3}^{+}}{\rightleftarrows}} E S S
$$

where the enzyme $E$ and the substrate $S$ bound together in an intermediate complex $E S$ that breaks down to product $P$ and free enzyme. In addition, the intermediate $E S$ reacts also with the substrate forming a dead-end complex ESS. Under the steady state assumption introduced by Haldane and Briggs [5], the concentrations of the species $E S, E S S$ are constant and in the product formation step the reverse rate 
of reaction is negligible with respect to the direct one $\left(k_{2}^{+}>>k_{2}^{-}\right)$. As a consequence of this assumption, the dynamics of mechanism (15) reduces to the two dimensional system (14) in which:

$$
V_{\text {max }}=k_{2}^{+} E_{0}, \quad K_{M}=\frac{k_{2}^{+}+k_{1}^{-}}{k_{1}^{+}}, \quad K_{i}=\frac{k_{3}^{-}}{k_{3}^{+}}
$$

In Fig. 1a the mechanism (15) is depicted under both a classical and a graphical description. The corresponding state-space equations will be described next, representing by means of the state vector $\left[x_{1}, x_{2}, x_{3}, x_{4}, x_{5}\right]$ the concentrations of the species $E, S, E S, E S S, P$.

2.4.2. The graph description: Let us consider the open system consisting of the mechanism (15) in which substrate and product are allowed to pass through the control volume as depicted in Fig. 1b, where $\mathcal{G}_{c}$ and $\mathcal{G}_{o}$ are the corresponding closed and open partitions of the graph. In agreement with (8):

$$
\begin{aligned}
& \dot{x}=\left(\begin{array}{cccccccc}
1 & 0 & 1 & 0 & 0 & 0 & 0 & 0 \\
1 & 0 & 0 & 1 & 0 & 1 & 0 & 0 \\
0 & 1 & 0 & 1 & 0 & 0 & 0 & 0 \\
0 & 0 & 0 & 0 & 1 & 0 & 0 & 0 \\
0 & 0 & 1 & 0 & 0 & 0 & 1 & 0
\end{array}\right) \cdot\left(\begin{array}{ccccc|c}
-k_{12} & k_{21} & 0 & 0 & 0 \\
k_{12} & -k_{21}-k_{23} & k_{32} & 0 & 0 & \\
0 & k_{23} & -k_{32} & 0 & 0 & 0 \\
0 & 0 & 0 & -k_{45} & k_{54} & \\
0 & 0 & 0 & k_{54} & -k_{54} & \\
0 & 0 & 0 & & & 0
\end{array}\right) \cdot\left(\begin{array}{c}
x_{1} \cdot x_{2} \\
x_{3} \\
x_{1} \cdot x_{5} \\
x_{2} \cdot x_{3} \\
x_{4} \\
\hline 0
\end{array}\right)+ \\
& \left(\begin{array}{llllllll}
1 & 0 & 1 & 0 & 0 & 0 & 0 & 0 \\
1 & 0 & 0 & 1 & 0 & 1 & 0 & 0 \\
0 & 1 & 0 & 1 & 0 & 0 & 0 & 0 \\
0 & 0 & 0 & 0 & 1 & 0 & 0 & 0 \\
0 & 0 & 1 & 0 & 0 & 0 & 1 & 0
\end{array}\right)\left(\begin{array}{c|ccc}
0 & & 0 & \\
\hdashline & -k_{68} & 0 & k_{86} \\
0 & -k_{78} & 0 \\
0 & k_{68} & -k_{86}-k 87
\end{array}\right) \cdot\left(\begin{array}{c}
0 \\
x_{2} \\
x_{5} \\
1
\end{array}\right)
\end{aligned}
$$

2.4.3. The classical kinetic description: The reaction rates for each step of the mechanism (15) are:

$$
W_{1}=k_{1}^{+} x_{1} x_{2}-k_{1}^{-} x_{3}, \quad W_{2}=k_{2}^{+} x_{3}-k_{2}^{-} x_{1} x_{5}, \quad W_{3}=k_{3}^{+} x_{3} x_{2}-k_{3}^{-} x_{4}
$$

and the dynamics, after adding the corresponding input and output terms (10) for the open system in Fig. $1 \mathrm{~b}$ is equivalent to (17) with $k_{78}=\phi, k_{68}=\phi, k_{86}=\phi x_{20}$ :

$$
\dot{x}=\left(\begin{array}{rrr}
-1 & 1 & 0 \\
-1 & 0 & -1 \\
1 & -1 & -1 \\
0 & 0 & 1 \\
0 & 1 & 0
\end{array}\right) \cdot\left(\begin{array}{l}
W_{1} \\
W_{2} \\
W_{3}
\end{array}\right)+\phi \cdot\left(\begin{array}{c}
0 \\
x_{2_{0}}-x_{2} \\
0 \\
0 \\
-x_{5}
\end{array}\right)
$$

2.4.4. The reaction simplex The mass conservation balances (11) for this case, in its closed form, are:

$$
\mathcal{C}_{X_{0}}^{0}=c_{X}^{T} x=x_{1}+x_{3}+x_{4}, \mathcal{C}_{S_{0}}^{0}=c_{S}^{T} x=x_{2}+x_{3}+2 x_{4}+x_{5}
$$

where $\mathcal{C}_{X}^{0}$ and $\mathcal{C}_{S}^{0}$ represent the concentrations of building blocks of the network, i.e., the $X$ and $S$ species. Vectors $c_{X}$ and $c_{S}$ can be grouped into the following matrix:

$$
C^{T}=\left(\begin{array}{lllll}
1 & 0 & 1 & 1 & 0 \\
0 & 1 & 1 & 2 & 1
\end{array}\right)
$$

which defines a basis for $\operatorname{ker} \mathcal{N}^{T}$ so that $C^{T} \mathcal{N}=0$. Consequently, each vector $C^{0}=\left(\mathcal{C}_{A}^{0}, \mathcal{C}_{B}^{0}\right)$ defines a specific reaction simplex $\Omega\left(x_{0}\right)$ for every initial condition $x_{0}$ satisfying $C^{0}=C^{T} x_{0}$. 


\section{The entropy balance and the dynamics of the network}

\subsection{The dissipative nature of reaction networks}

According to the second law of thermodynamics isolated systems evolve to equilibrium through irreversible processes that produce entropy, being the rate of entropy production a way to quantify dissipation. In exploring the stability of the equilibrium manifold, we follow [4] and define an entropy-like expression as the negative of the function:

$$
S(x)=\sum_{i=1}^{n} x_{i} \cdot\left(\ln x_{i}-1\right)
$$

that coincides with the Lyapunov function used in [3] for the demonstration of the so-called Deficiency Zero Theorem. The right hand side term in (9) is Lipschitz continuous, as discussed in [1], this implies that for any arbitrary reference $x_{1}$ there exists a nonnegative function $L_{\lambda}\left(x, x_{1}\right)$ associated to a constant $\lambda \geq 0$, such that the following relation holds:

$$
\left[\mu(x)-\mu\left(x_{1}\right)\right]^{T} \mathcal{N} \cdot W(x)+L_{\lambda}\left(x, x_{1}\right)=\lambda\left[\mu(x)-\mu\left(x_{1}\right)\right]^{T}\left(x-x_{1}\right)
$$

where $\mu=\nabla_{x} S=\left[\begin{array}{lll}\ln x_{1} & \ldots & \ln x_{n}\end{array}\right]^{T}$. Systems which, in addition, satisfy that $L_{0}\left(x, x_{1}\right) \geq 0$, are referred as purely dissipative in [1]. Closed reaction networks are a class of purely dissipative systems for a state $x_{1}$ being the equilibrium reference. This can be easily shown by noting that for $\lambda=0$ and $x_{1}=x^{*}$ in (23) we have:

$$
L_{0}\left(x, x^{*}\right)=-\left(\mu-\mu^{*}\right)^{T} \sum_{j=1}^{r} \nu_{j} W_{j}=\sum_{j=1}^{r} \ln \frac{W_{j}^{+}}{W_{j}^{-}} \cdot\left(W_{j}^{+}-W_{j}^{-}\right)
$$

Since each term at the right hand side of $(24)$ is non-negative we conclude that $L_{0}\left(x, x^{*}\right) \geq 0$. In order to derive the stability conditions for closed reaction networks we define a positive definite and convex function $B(x)$, constructed as the difference between $S(x)$ and its supporting hyperplane at the equilibrium reference $x^{*}$ :

$$
B\left(x, x^{*}\right)=\sum_{i=1}^{n} x_{i}\left(\ln \frac{x_{i}}{x_{i}^{*}}-1\right)+x_{i}^{*}
$$

Taking the time-derivative $B\left(x, x^{*}\right)$ along (25) and using (23) with $\lambda=0$ we obtain:

$$
\dot{B}=\left(\mu-\mu^{*}\right)^{T} \sum_{j=1}^{r} \nu_{j} W_{j}=-L_{0}\left(x, x^{*}\right)
$$

Since by (24) $L_{0} \geq 0$ we have that $\dot{B} \leq 0$. It is easy to check that $B$ is bounded from below, globally convex and it has a global minimum in $x=x^{*}$. Consequently, $B$ is a legitimate Lyapunov function which ensures the structural stability of the reaction network at the equilibrium reference.

\subsection{The non-dissipative contributions}

The material throughput flow induce non-dissipative contributions to the system, by adding a new term in the entropy balance: the entropy flux. In our formalism, the entropy balance is obtained by computing the time derivative of $B$ as defined in (26) along (10):

$$
\dot{B}=\left(\mu-\mu^{*}\right)^{T} \sum_{j=1}^{r} \nu_{j} W_{j}+\left(\mu-\mu^{*}\right)^{T} \phi\left(x^{0}-x\right)
$$


The first term in the right hand side of $(27)$ corresponds to the entropy production $\left(P_{B}\right)$, while the second term is the entropy flux $\left(\Phi_{B}\right)$ that may compensate or even override the natural entropy dissipation, thus undermining the inherent global stability of the system. To illustrate this in the Michaelis Menten example, the time derivative of the function $B(27)$ and the corresponding trajectories in the phase space (19) are depicted in Fig. 2 for increasing values of the parameter $\phi$. In Fig. $2 \mathrm{~b}$ three steady states can be observed, the unstable one corresponding to the region of the state space for which the value of $\dot{B}$ is positive in Fig. 2f. It seems therefore crucial to act on the non dissipative contributions by appropriate control configurations in order to stabilize reaction networks.

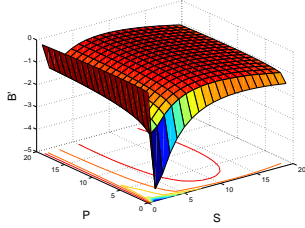

(a)

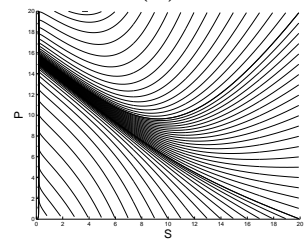

(e)

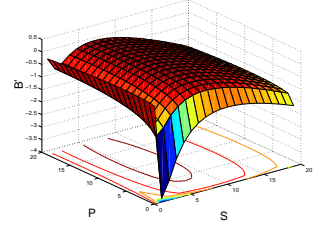

(b)

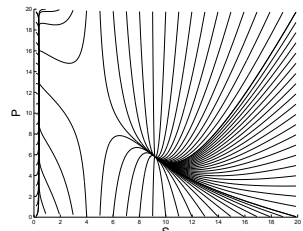

(f)

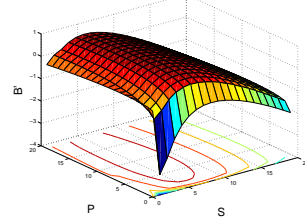

(c)

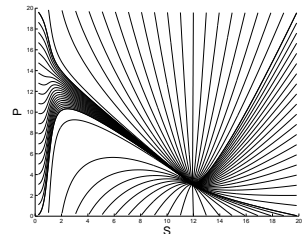

(g)

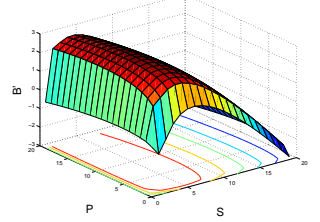

(d)

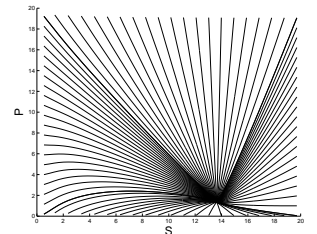

(h)

Figure 2. From left to right $\dot{B}$ and the corresponding trajectories in the phase space for increasing values of $\phi$

\section{Biochemical networks stabilization through nonlinear control}

\subsection{Passivity and its application in nonlinear control}

In the framework of nonlinear control theory, reaction systems will be represented in the following general input-affine state-space form with $m$ inputs $u \in U=\mathbb{R}^{m}$ and $p$ outputs $y \in Y=\mathbb{R}^{p}$ :

$$
\begin{aligned}
\dot{x} & =f(x)+G(x) u \\
y & =h(x)
\end{aligned}
$$

Where the state vector $x=\left[x_{1}, \ldots, x_{n}\right]^{T}$ is assumed to belong to an open set $X$ of $\mathbb{R}^{n}, f \in \mathbb{R}^{n} \mapsto \mathbb{R}^{n}$, $G(x) \in \mathbb{R}^{n \times m}$ for all $x \in X, h \in \mathbb{R}^{n} \mapsto \mathbb{R}^{p}$, and $f(0)=0$.

A system of the form (28) with $p=m$ is said to be passive if there exists a $C^{0}$ nonnegative function $V: X \rightarrow \mathbb{R}$, called the storage function, such that for all $u \in U, x\left(t_{0}\right) \in X, t_{1} \geq t_{0}$

$$
V\left(x\left(t_{1}\right)\right) \leq V\left(x\left(t_{0}\right)\right)+\int_{t_{0}}^{t_{1}} y^{T}(t) u(t) d t, \forall t>0
$$

or equivalently:

$$
\dot{V}(t) \leq y^{T}(t) u(t), \forall t>0
$$

Inequality (29) originally comes from the theory of electrical circuits and expresses the fact that the stored energy $V$ at any future time $t_{1}$ is at most equal to the sum of the stored energy at the present 
time $t_{0}$ plus the total externally supplied energy $\int_{t_{0}}^{t_{1}} y^{T}(t) u(t) d t$. This principle can be generalized for other nonlinear systems where $V$ is not necessarily energy but a suitable abstract quantity. If $V$ has appropriate geometry (e.g. it is bounded from below and proper), then it can be used as a Lyapunov function for stability analysis of the uncontrolled (open-loop) or controlled (closed-loop) system. Passive systems have many interesting and advantageous properties from a control point of view, but we will emphasize here only two of them. Firstly, if $V$ can be used as a Lyapunov function, then passive systems can be globally asymptotically stabilized with a feedback $u=-\phi(y)$ where $\phi(0)=0$ and $y^{T} \phi(y)>0$ $\forall y \neq 0$. Secondly, if a storage function candidate is previously known for the uncontrolled system, i.e. $\frac{\partial V}{\partial x} f(x) \leq 0$ and the input is given, then the system will be passive if we choose the output in the following way: $y^{T}=\frac{\partial V}{\partial x} G(x)$. (For the proofs and details see, e.g. [12]).

It is visible that (27) expresses a passivity relation with $V=B, y=\mu-\mu^{*}$ and $u=\phi\left(x^{0}-x\right)$.

\subsection{Stabilization in the state space}

In this section we propose a meaningful way to modify the entropy flux by means of a nonlinear controller to stabilize reaction networks with $u=x^{0}$, provided that observability and controllability conditions are satisfied [9].

A simple way of stabilizing steady states is summarized in the following proposition:

Proposition 2. Any stationary solution $x^{*}$ of (10) can be rendered exponentially stable by a control law of the form $x^{0}=x^{0 *}-\omega\left(x-x^{*}\right)$ with $\omega \geq(\lambda-\phi-\alpha) / \phi>0$ and $\alpha<0$

Proof: First, let us construct a $B$ function as in (25) with respect to the stationary solution $x^{*}$ associated to the input concentration vector $x^{0 *}$. Defining $\bar{x}=x-x^{*}, \bar{x}^{0}=x^{0}-x^{0 *}$ and computing the time derivative of $B$ along (10) we get:

$$
\dot{B}=\left(\mu-\mu^{*}\right)^{T} \mathcal{N}\left(W(x)-W\left(x^{*}\right)\right)+\phi\left(\mu-\mu^{*}\right)^{T}\left(\bar{x}^{0}-\bar{x}\right)
$$

Since $B\left(x, x^{*}\right)$ is convex, we also have the following inequality:

$$
B\left(x, x^{*}\right) \geq\left(\mu-\mu^{*}\right)^{T} \bar{x}
$$

Substituting (23) and the control law in (30) we obtain:

$$
\dot{B}=-L_{\lambda}+(\lambda-\phi-\phi \omega)\left(\mu-\mu^{*}\right)^{T} \bar{x}
$$

Finally, since $L_{\lambda}>0$, and $B$ satisfies (31), equation (32) becomes $\dot{B} \leq \alpha B$ and the result follows.

\subsection{Stabilization and passivity in the reaction space}

4.2.1. The potential structure of the reaction space: By introducing a nonlinear coordinates-transformation, we will demonstrate next that a complex reaction network possesses an underlying potential structure on a state space homeomorphic to the concentration space, that will be referred to as the reaction space. Let us define the following new coordinates:

$$
z_{j}=\ln \frac{p_{j}}{q_{j}} \quad \text { for } j=1, \ldots, r
$$

with $p_{j}$ and $q_{j}$ being the direct and reverse rates associated to the reaction step $j\left(W_{j}^{+}\right.$and $\left.W_{j}^{-}\right)$. In the new variables, equation (2) becomes:

$$
W_{j}=p_{j}-q_{j}=q_{j}\left(e^{z_{j}}-1\right)
$$


The right hand side of Eq. (24) can then be transformed through appropriate manipulations into the form:

$$
\ell(z, q)=-\sum_{j=1}^{r} z_{j} q_{j}\left(e^{z_{j}}-1\right)=-z^{T} W
$$

Function $\ell$ can be easily connected with the so called dissipation function [2] as it is the product of thermodynamic fluxes (reaction rates) and thermodynamic forces (chemical affinities). In this way, it seems natural to explore the properties of chemical reaction network dynamics in the reaction space defined by $z$-variables. For that purpose, let us introduce the following notations:

$$
S=\mathcal{N}^{T} \Gamma \mathcal{N}, \quad \Gamma=\operatorname{diag}\left[\frac{1}{x_{1}}, \ldots, \frac{1}{x_{i}}, \ldots \frac{1}{x_{n}}\right]
$$

The particular structure of the elements of the reaction vector (34) suggests the definition of the following potential:

$$
H(z)=\sum_{j=1}^{r}\left(e^{z_{j}}-z_{j}\right)-1
$$

and re-write the vector of reaction rates as:

$$
W=F(q) H_{z}
$$

where $F(q)=\operatorname{diag}\left[q_{1}, \ldots, q_{j}, \ldots, q_{r}\right]$ is a positive definite diagonal matrix and $H_{z}$ represents the gradient of (37). Note that by construction, the potential $H$ is convex and positive definite everywhere except at the equilibrium point $z^{*}=0$, where $H(0)=0$. Defining $J=S F(q)$ we obtain the following potential dynamic description:

$$
\dot{z}=-J H_{z}
$$

where $H_{z}$ is the gradient of $H$ (written as a column vector). It is clear from (39) that if $J$ is positive definite, then $H$ is decreasing in time and the global asymptotic stability of the reaction system in the reaction space can be concluded (with $H$ as a Lyapunov function). It is interesting to note that in this case, the dynamics in the reaction space gives a so-called generalized Hamiltonian system [12]. However, it can be shown that there are such reaction networks, where $J$ is not globally positive definite.

4.2.1. Stabilization in the reaction space: The dynamic evolution of an open reaction network (10) in the reaction space is described as:

$$
\dot{z}=-J H_{z}-\mathcal{N}^{T} \Gamma \phi\left(x^{0}-x\right)
$$

where $J \in \mathbb{R}^{r \times r}$ is a positive definite matrix. The potential structure discussed above allows us to establish a straightforward connection with passivity, summarized next:

Proposition 3. If $J^{T}+J$ is globally positive definite, then the system (40) endowed with the function $H$ (37) is passive with $y=H_{z}$ and $u=\mathcal{N}^{T} \Gamma \phi\left(x-x^{0}\right)$.

Proof: We compute the time derivative of the potential (37) along the trajectories defined by (40) using the definitions of $y$ and $u$ to obtain:

$$
\dot{H}=-H_{z}{ }^{T} J H_{z}-H_{z}{ }^{T} \mathcal{N}^{T} \Gamma \phi\left(x^{0}-x\right)=-H_{z}{ }^{T} J H_{z}+y^{T} u
$$

It clearly follows from (41) that the passivity inequality (29) is valid in the reaction space in this case.

Remark 1. If $J^{T}+J$ is not globally positive definite, then open reaction networks can be globally stabilized in the reaction space with control Lyapunov function $H$ by nonlinear control laws of the form:

$$
u=\mathcal{N}^{T} \Gamma(\mathcal{N} F+K \Gamma \mathcal{N}) y
$$


where $K \in \mathbb{R}^{n \times n}$ is an appropriate (and possibly state dependent) matrix. Applying the feedback (42) the time-derivative of $H$ can be calculated as

$$
\dot{H}=y^{T} \mathcal{N}^{T} \Gamma K \Gamma \mathcal{N} y
$$

which is negative if $K$ is negative definite (or zero if $K$ is skew-symmetric).

To illustrate this in the Michaelis Menten example, starting from the definition of the $p$ and $q$ variables and according with (18), the new coordinates are:

$$
z_{1}=\ln \frac{k_{1}^{+} x_{1} x_{2}}{k_{1}^{-} x_{3}}, \quad z_{2}=\ln \frac{k_{2}^{+} x_{3}}{k_{2}^{-} x_{1} x_{5}}, \quad z_{3}=\ln \frac{k_{3}^{+} x_{3} x_{2}}{k_{3}^{-} x_{4}}
$$

Taking the expression of $y$ in Proposition 3 and the proposed control law $u$, the convergence of the kinetic system to the steady state can be significantly improved by using e.g. the following feedback gain matrix in (42): $K=-0.1 \cdot I^{5 \times 5}$, where $I$ denotes the unit matrix.

\section{Conclusions}

In this work, we have first shown how under some assumptions and the proper control volume selection, a general class of biochemical reaction networks fits into two equivalent descriptions: one coming from the graph of a reaction network and the other derived from the classical kinetic formalism. As a first conclusion, $C N R T$ stability results directly apply to such general class of biochemical systems. In addition, both representations allow us to rigorously apply concepts and tools from irreversible thermodynamics in the dynamic analysis and stabilization of biochemical reaction networks, by stating an entropy balance over compact regions of the state space, the so called reaction simplices. We have shown by means of thermodynamic arguments that closed reaction networks are purely dissipative systems, then concluding that dynamical complexity is associated to the entropy flux through the boundary. Based on this physical insight, the entropy flux is meaningfully manipulated via the nonlinear control laws proposed, achieving the efficient stabilization of arbitrary steady states in biochemical networks. We have demonstrated as well the inner underlying potential structure of this general class of biochemical networks, and then passivity theory has been applied to develop suitable nonlinear control laws in order to ensure stability in the reaction space. The explored potential structure and the passivity framework may facilitate the subsequent extension of our results to higher level structures of interconnected systems and signals, such as populations of coupled oscillators [8]. As another direction of future work, the graphical description that we have proposed might be employed to derive observability and controllability results exploiting its special structure.

\section{Acknowledgements}

The authors acknowledge financial support received from the Spanish Government (MCyT Projects PPQ2001-3643 \& DPI2004-07444-C04-03) and Xunta de Galicia (PGIDIT02-PXIC40209PN). This research was partially supported by the Hungarian grants no. T042710, F046223, which are gratefully acknowledged. The second author is a grantee of the Bolyai János Research Scholarship of the Hungarian Academy of Sciences.

\section{References}

[1] A. A. Alonso and E. Ydstie. Process systems, passivity and the second law of thermodynamics. Computers and Chemical Engineering, 20:S1119-S1124, 1996. 
[2] Y. Demirel and S.I. Sandler. Thermodynamics and bioenergetics. Biophysical Chemistry, 97:87-111, 2002.

[3] M. Feinberg. Lectures on chemical reaction networks. Notes of lectures given at the Mathematics Research Center, University of Wisconsin, 1979.

[4] A. N. Gorban, I.V. Karlin and A.Y. Zinovyev, Invariant grids for reaction kinetics, Physica A, 2004, 33:106-154.

[5] G. E. Briggs, J. B. S. Haldane, A note on the kinetics of enzyme action. Biochem. J. 19 (1925) 338-339.

[6] J. Gunawardena. Chemical Reaction Network Theory for In-silico Biologists. Lecture notes. Bauer Center for Genomics Research. Harvard University, 2003.

[7] J. Hasty, D. McMillen and J. J. Collins. Engineered gene circuits. Nature 420: 224-230, 2002.

[8] J.C. Leloup and A. Goldbeter. Modeling the mammalian circadian clock: Sensitivity analysis and multiplicity of oscillatory mechanisms. Journal of Theoretical Biology, 230:541-562, 2004.

[9] E. Sontag, A. Kiyatin and B. N. Kholodenko. Inferring dynamic architecture of cellular networks using time series of gene expression, protein and metabolite data. Bioinformatics, 20(12):1877-1889, 2004.

[10] S.H. Strogatz. Exploring complex networks, Nature,410:268-276,2001.

[11] R. Thomas and M. Kaufman. Multistationarity, the basis of cell differentiation and memory. I. Structural conditions of multistationarity and other nontrivial behaviour. Chaos, 11:170-179, 2001.

[12] Van der Schaft, A., $L_{2}$-Gain and Passivity Techniques in Nonlinear Control, Springer, 2000 